\title{
Infrared absorption and Raman scattering of $\mathrm{OH}^{-}$and $\mathrm{OD}^{-}$stretching-mode vibrations in alkali fluorides: Isolated defects
}

\author{
E. Gustin, A. Bouwen, and D. Schoemaker \\ Department of Physics, University of Antwerp (UIA), Universiteitsplein 1, B-2610 Antwerpen, Belgium \\ C. P. An and F. Luty \\ Department of Physics, University of Utah, Salt Lake City, Utah 84112
}

(Received 28 June 1999)

\begin{abstract}
The stretching-mode (SM) of $\mathrm{OH}^{-}$and $\mathrm{OD}^{-}$defects in $\mathrm{LiF}, \mathrm{NaF}$, and $\mathrm{KF}$ hosts has been studied with Raman scattering and Fourier transform infrared (FTIR) absorption spectroscopy, at temperatures from $4 \mathrm{~K}$ to $500 \mathrm{~K}$. In all three hosts the $\langle 111\rangle$ orientation of the $\mathrm{OH}^{-}$molecular axis is confirmed both by polarized Raman spectra and by stress-induced FTIR dichroism. The latter technique also yields the values of the disc-shaped elastic dipole tensor, aligning the $\mathrm{OH}^{-}$parallel to an applied $\langle 111\rangle$ uniaxial stress with a Curie law. Accurate band-shape analysis of low-temperature FTIR absorption and $T_{2 g}$ Raman spectra yields for low concentrations of $\mathrm{OH}^{-}$in $\mathrm{LiF}$ and $\mathrm{KF}$ consistent values of their nearest-neighbor reorientational tunneling parameters $\left(\Delta=1.2\right.$ and $0.6 \mathrm{~cm}^{-1}$, respectively). For $\mathrm{OH}^{-}$in $\mathrm{NaF}$ and for $\mathrm{OD}^{-}$in all three hosts spectral substructure from tunneling is undetectable in the narrow Lorentzian-shaped SM lines. The strong temperature broadening that occurs for the SM absorption and $T_{2 g}$ Raman bands and the smaller broadening of the $A_{1 g}$ line can be fitted with models for the dephasing of the transition by phonons, considering the presence of isotropic and anisotropic dephasing components. In spite of the very different amplitudes of the $\mathrm{OH}^{-}$and $\mathrm{OD}^{-} \mathrm{SM}$ vibrations, their relative infrared absorption and Raman intensities are found to be very similar; the only exception is an extremely weak oscillator strength of the $\mathrm{OD}^{-}$absorption in $\mathrm{KF}$.
\end{abstract}

\section{INTRODUCTION}

The extensive studies of $\mathrm{OH}^{-}$defects in alkali halides during the past 35 years have mostly focused on alkalichloride, -bromide, and -iodide crystals. ${ }^{1}$ In all these hosts, substitution of a small $\mathrm{OH}^{-}$molecular ion on larger $\mathrm{Cl}^{-}$, $\mathrm{Br}^{-}$, or I- lattice sites leads to sizable off-center shifts, large (molecular + displacement) electric dipoles, and "cigarshaped" elastic dipole tensors of either $\langle 100\rangle$ or $\langle 110\rangle$ symmetry. Orientational tunneling of these systems involves complicated angular and translational motion of the molecule. The theoretical description includes a renormalization of its tunneling parameter $\Delta$ to a much smaller value, taking care of the reorientation of the strong gerade and ungerade distortions of the surrounding lattice. ${ }^{2}$ This sizable "dressing effect" obscures (or may even invert) the large difference $\Delta\left(\mathrm{OH}^{-}\right)>\Delta\left(\mathrm{OD}^{-}\right)$expected for the tunneling of the "bare molecule."

In distinct contrast to these systems, the "egg-shaped" $\mathrm{OH}^{-}$molecule fits very tightly into the $\mathrm{F}^{-}$lattice site of alkali-fluorides. Regarding an $\mathrm{OH}^{-}$ion simplified as an " $\mathrm{F}^{-}$ ion with one of its protons pulled out by $\sim 1 \AA$," illustrates its being a bit longer and slimmer, compared to an $\mathrm{F}^{-}$ion. Due to this misfit it has been often suspected that reorientational tunneling of $\mathrm{OH}^{-}$may not be possible in alkali fluorides. This appeared to be supported experimentally by negative results of attempted paraelectric resonance ${ }^{3}$ and electrodichroism experiments ${ }^{4}$ in various alkali fluorides. Two encouraging positive results on $\mathrm{LiF}: \mathrm{OH}^{-}$with lowtemperature heat conductivity ${ }^{5}$ and dielectric experiments, ${ }^{6}$ indicating the presence of defects with sizable $1 \sim 2 \mathrm{~cm}^{-1}$ tunneling splitting, remained "singularities;" they were not tested with other techniques, or extended by studies of the defect symmetry, $\mathrm{OH}^{-} \rightarrow \mathrm{OD}^{-}$isotope or alkali fluoride host material variation.

This uncertainty about tunneling and reorientation of $\mathrm{OH}^{-}$molecules in alkali fluorides changed drastically two years ago with a paper by two of the present authors (CPA and FL). ${ }^{7}$ Stress dichroism of the stretching-mode (SM) absorption of $\mathrm{OH}^{-}$and $\mathrm{OD}^{-}$molecules in $\mathrm{LiF}$ and $\mathrm{NaF}$ showed clearly that these defects can be aligned paraelastically at lowest temperatures with uniaxial stress $S_{111}$ and characterized by "disk-shaped elastic tensors." Moreover, the SM absorption of $\mathrm{OH}^{-}$in LiF showed clearly a doubleband structure, caused predominantly by $70^{\circ}$ tunneling of the $\langle 111\rangle$ oriented molecules, and yielding a tunneling splitting parameter of $\Delta=1.2 \mathrm{~cm}^{-1}$ for both the ground and excited states of the SM transition. ${ }^{7}$ The unresolved estimated tunneling splitting of $\mathrm{OD}^{-}$in both $\mathrm{LiF}$ and $\mathrm{NaF}$ hosts was found to be considerably smaller than that of $\mathrm{OH}^{-}$.

We extend this initial work of Ref. 7 in two important directions: adding a new host material KF and an important experimental technique, nonresonant Raman scattering, to the Fourier transform infrared (FTIR) absorption study of the $\mathrm{OH}^{-}$and $\mathrm{OD}^{-}$stretching modes. First these two FTIR and Raman approaches probe different physical properties of the system: SM modulation of the electric dipole vectors vs SM modulation of the electronic polarizability tensors of the molecular defects. This often leads to results that can be used for complementary interpretations. Polarized Raman scattering of properly chosen symmetries of the incident and scat- 
tered light, can for a totally unaligned molecular defect system determine the orientation $(\langle 100\rangle,\langle 111\rangle$, or $\langle 110\rangle)$ of the molecules. We use this "behavior type (BT) method" for our studied crystal-defect system ${ }^{8}$ (see Sec. III A) and compare the results to the ones obtained by the infrared (IR) absorption dichroism for uniaxial stress-aligned molecular defects (see Sec. III B). For systems with sizable tunneling splitting, the expected spectral substructure of IR or Raman SM transitions is quite different due to different selection rules between the gerade and ungerade tunneling states. We present results obtained in $\mathrm{LiF}: \mathrm{OH}^{-}$and $\mathrm{KF}: \mathrm{OH}^{-}$and discuss attempts to get them in agreement to each other and to the underlying tunneling model in Sec. III C. This latter attempt is particularly challenging for the newly studied $\mathrm{KF}: \mathrm{OH}^{-}$system, which-in spite of its determined $\langle 111\rangle$ symmetry-is found to be not in agreement with a simple model of predominant nearest-neighbor $70^{\circ}$ tunneling. Addition of Raman has added to the single SM half-width of IR absorption two new ones, observed half-width (HW) values for parallel $\left(A_{1 g}\right)$ or perpendicular $\left(T_{2 g}\right)$ light scattering. Their observed broadening under temperature increase (up to $500 \mathrm{~K}$ ) is presented, and tried to be interpreted by assumed dephasing mechanisms in Sec. III D. Similarly, we treat in Sec. III E strong temperature dependence of SM line positions, observed by IR absorption, $A_{1 g}$ and $T_{2 g}$ Raman measurements. Finally, in Sec. III F results of the SM IR absorption and Raman strength of $\mathrm{OH}^{-}$and $\mathrm{OD}^{-}$in the three hosts are presented and discussed.

\section{EXPERIMENTAL DETAILS}

The crystals were grown with the Kyropolous technique at the University of Utah from a melt of ultrapure powder of host material, doped with a proper alkali-hydroxide. Acid titration of measured samples was used to determine the $\mathrm{OH}^{-}$and $\mathrm{OD}^{-}$concentrations. We then used these to determine the absorption strength per unit of concentration, as listed in Table II. By varying the defect concentration and comparing the absorption spectra, we were able to select concentrations at which the interactions between defects are negligible. At higher temperatures $(T \geqslant 150 \mathrm{~K})$ high $\mathrm{OH}^{-} / \mathrm{OD}^{-}$concentrations could be used because the thermal broadening of linewidths is larger than the inhomogeneous one due to the defect interaction and background strain. We obtained the same band shapes in the temperature region for high and low concentrations.

The infrared absorption spectra were measured with a Bruker IFS88 FTIR spectrometer with a resolution of about $0.1 \mathrm{~cm}^{-1}$. The samples were placed in a helium cryostat, that allowed temperature variations between $4 \mathrm{~K}$ and $350 \mathrm{~K}$.

The Raman scattering experiments were performed with a Dilor XY-800 Raman Spectrometer, equipped with a Wright Instruments nitrogen-cooled $1200 \times 300$-pixel CCD detector. Measurements at temperatures between $4 \mathrm{~K}$ and $150 \mathrm{~K}$ were done in a helium-flow cryostat, and measurements between $130 \mathrm{~K}$ and $500 \mathrm{~K}$ in a cryostat cooled by nitrogen gas. For most measurements the $514.53 \mathrm{~nm}$ line of a Spectra Physics $2020 \mathrm{Ar}^{+}$-laser was used as excitation source. This yields a spectral resolution of $1.7-1.5 \mathrm{~cm}^{-1}$ in the low-dispersion mode of the spectrometer, and of $0.3 \mathrm{~cm}^{-1}$ in the highresolution mode. All Raman scattering experiments were

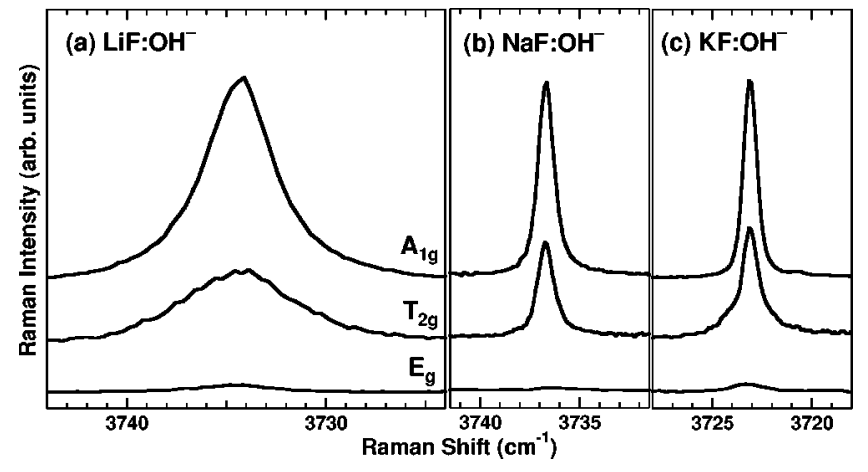

FIG. 1. Relative intensities of the $A_{1 g}, E_{g}$, and $T_{2 g}$ Raman scattering of the $\mathrm{OH}^{-}$stretching mode in $\mathrm{LiF}$ (a), NaF (b), and KF (c), obtained from polarized Raman measurements at $6.2 \mathrm{~K}$.

performed in a $90^{\circ}$ scattering geometry. Samples cleaved along (100) surfaces were used to record $\langle 100,100\rangle$ (or $A_{1 g}$ $\left.+E_{g}\right)$ and $\langle 100,010\rangle\left(\frac{1}{2} T_{2 g}\right)$ spectra. Samples polished to have two (110) surfaces gave us $\langle 110,1 \overline{1} 0\rangle\left(\frac{3}{4} E_{g}\right)$ and $\langle 110,110\rangle\left(A_{g}+\frac{1}{4} E_{g}+\frac{1}{2} T_{2 g}\right)$ spectra.

Note that for the symmetry notations $E_{g}$ and $T_{2 g}$ we are using the convention of Ref. 8, which differs from the one used by Ref. 9 by factors 4/3 and 2, respectively.

\section{EXPERIMENTAL RESULTS AND DISCUSSION}

\section{A. Polarized Raman scattering: Defect symmetry}

We have verified the molecular orientation of $\mathrm{OH}^{-}$and $\mathrm{OD}^{-}$in alkali-fluoride hosts, determined in a previous work by measurements of the elastic dichroism of SM absorption, ${ }^{7}$ with polarized Raman scattering experiments. In spite of an equal distribution of the microscopic polarizability tensors of the defects over the possible orientations, the resulting macroscopic polarized Raman response will be different for $\langle 100\rangle,\langle 111\rangle$, or $\langle 110\rangle$ orientations of the individual molecules. It is often complicated to extract symmetry information from a set of Raman spectra. A quantitative approach called the behavior type method ${ }^{10}$ has been applied to diatomic molecules by Fleurent et al. ${ }^{8}$

The three symmetry components of the Raman spectra at $6.2 \mathrm{~K}$ for $\mathrm{OH}^{-}$in three hosts are displayed in Fig. 1. The $\langle 110,1 \overline{1} 0\rangle$ spectrum is very weak, only a few percent of the $\langle 100,100\rangle$ intensity. This weak signal may be due to the imperfection of the polarizer, the focusing of the laser beam, and small errors in orientation. Therefore we can say that the $E_{g}$ spectrum is zero within the experimental limits. The absence of an $E_{g}$ spectrum and the presence of a sizable $T_{2 g}$ spectrum are compatible with an $\langle 111\rangle$ orientation of the defects, but not with a $\langle 100\rangle$ or $\langle 110\rangle$ orientation. ${ }^{8}$

In case the thermal reorientation becomes sufficiently rapid at high temperatures, the system may approach the free rotor behavior, resulting in a reduction of the $T_{2 g}$ intensity and the appearance of an $E_{g}$ spectrum. For the case of $\mathrm{NaCl}: \mathrm{OH}^{-}$this has indeed been observed. ${ }^{9}$ We found no $E_{g}$ spectrum in $\mathrm{NaF}$ at room temperature, yet in all three host materials the ratio of the $T_{2 g} / A_{1 g}$ intensities decreases when the temperature is increased above $120 \mathrm{~K}$. This may indicate some increase in angular motion. However, the impurity is 


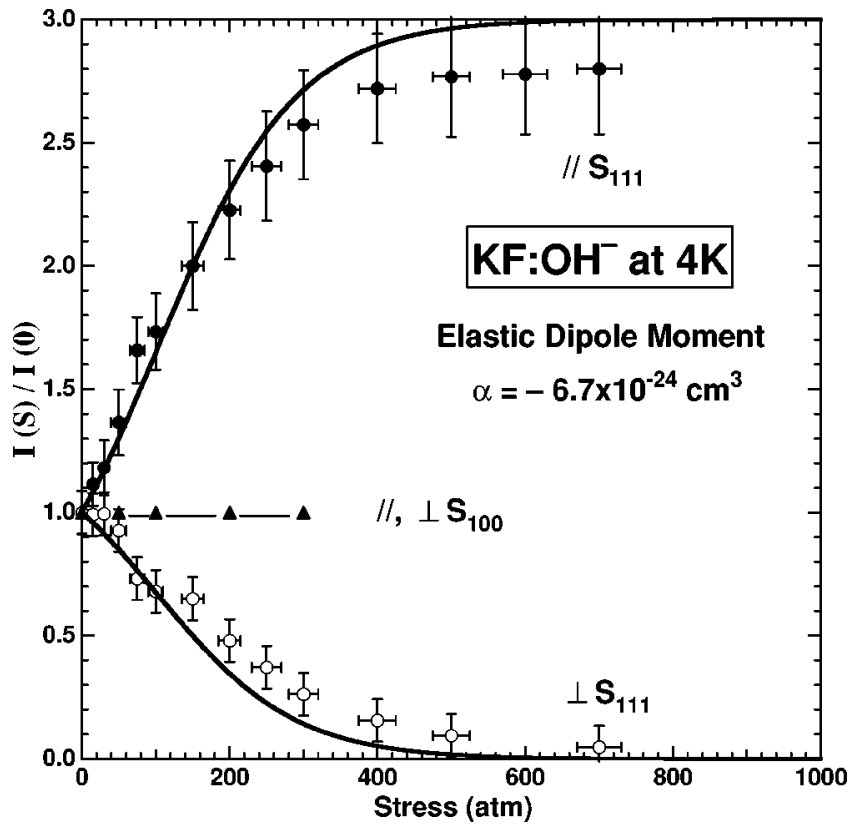

FIG. 2. Integrated $\mathrm{OH}^{-}$absorption ratio $I(S) / I(0)$ in $\mathrm{KF}$ host under variation of $S$, measured parallel and perpendicular polarized to the applied stress $S$. The curves are calculated and fitted for the $\langle 111\rangle$ elastic-dipole model as described in text.

not expected to come close to the behavior of a free rotor, because the barriers for reorientation of the $\mathrm{OH}^{-}$are quite high.

The Raman spectra of $\mathrm{NaF}: \mathrm{OD}^{-}$and $\mathrm{KF}: \mathrm{OD}^{-}$also indicate, by their $T_{2 g} / E_{g}$ behavior, an $\langle 111\rangle$ orientation of the defect. The $E_{g}$ spectrum of $\mathrm{LiF}: \mathrm{OD}^{-}$was not recorded.

\section{B. Stress alignment: Elastic dipole tensor}

The molecular defects introduce sizable anisotropic elastic lattice deformations, which can be represented by the "elastic dipole tensors." 11 The integrated SM absorption changes $I(S) / I(0)$ induced by a stress $S$ of a particular symmetry correspond directly to the paraelastic alignment under stress of the defects. ${ }^{12}$ For $\mathrm{OH}^{-}$and $\mathrm{OD}^{-} \mathrm{LiF}$ and $\mathrm{NaF}$, elastic dichroism measurements indicating a $\langle 111\rangle$ oriented disk-shape elastic dipole moment were already reported in a previous work. ${ }^{7}$ To make the comparison complete for all three hosts, we now present the elastic dichroism of the IR SM absorption in KF. Figure 2 summarizes this stress-optical dichroism for $\mathrm{OH}^{-}$in $\mathrm{KF}$ as a function of $S$ at $4.2 \mathrm{~K}$, for stress applied in $\langle 100\rangle$ and $\langle 111\rangle$ directions.

The observed absence of any dichroism for $S_{100}$ and its obtained size and sign for $S_{111}$ show that $\mathrm{OH}^{-}$molecular ions in KF also form $\langle 111\rangle$ oriented disk-shaped elastic dipole tensors. The measured $I(S) / I(0)$ can be fitted very well with the calculated relations, Eqs. (1) and (2) in Ref. 7, yielding the negative elastic dipole factor $\alpha_{111}$ to be -6.7 $\times 10^{-24} \mathrm{~cm}^{3}$.

As far as could be observed the integrated stress dichroism of the $\mathrm{OD}^{-}$in $\mathrm{KF}$ behaves similarly. The $\mathrm{OD}^{-} \mathrm{SM}$ absorption is very weak and narrower than the resolution of the instrument, and an accurate analysis was not possible.

\section{Spectral substructure: Reorientational tunneling and half-width}

\section{Reorientational tunneling}

The reorientational tunneling between eight equivalent $\langle 111\rangle$ potential wells has been studied for the $\mathrm{LiF}^{-} \mathrm{OH}^{-}$system by measuring and analyzing the IR SM absorption spectra in Ref. 7. The presence of reorientational tunneling, through possible $70^{\circ}, 110^{\circ}$, and $180^{\circ}$ reorientation angles, splits the eightfold degenerate localized states into tunneling states with $A_{1 g}, T_{1 u}, T_{2 g}$, and $A_{2 u}$ symmetries. ${ }^{13}$ The $T_{1 u}$ and $T_{2 g}$ levels are threefold degenerate. If $70^{\circ}$ tunneling is dominant, the level splittings are equidistant. In the simplest case, the level spacing can be assumed to be identical in the ground and excited state of the SM vibration.

In absorption only the $g \leftrightarrow u$ transitions (except $\left.A_{1 g} \leftrightarrow A_{2 u}\right)$ are allowed, and the tunneling substructure of $\mathrm{SM}$ transition from $\mathrm{v}=0$ to 1 results in the appearance of two components at frequencies $\omega_{S M}+\Delta$ and $\omega_{S M}-\Delta$. In Raman scattering only $g \leftrightarrow g$ and $u \leftrightarrow u$ transitions are allowed, with additional restrictions imposed by the symmetry of the system and the polarization. In the $T_{2 g}$ Raman spectra three components appear, at frequencies $\omega_{S M}-2 \Delta, \omega_{S M}$, and $\omega_{S M}+2 \Delta$. In the $A_{1 g}$ spectrum there is only a line at $\omega_{S M} \cdot{ }^{14,15}$

Considering the selection rules for IR absorption transitions, the SM absorption of the $\mathrm{LiF}: \mathrm{OH}^{-}$system at $4.2 \mathrm{~K}$ can be decomposed into Stokes and anti-Stokes shifted Lorentzians of the same half-width, ${ }^{16}$ as shown on Fig. 3(a). The vertical bars, indicating the expected amplitude of the components on the basis of a Boltzmann distribution, agree closely with the observed intensities. In Ref. 7 their splitting has been studied under temperature and stress variations, yielding the energy-splitting parameter $\Delta=1.2 \mathrm{~cm}^{-1}$ with predominant $70^{\circ}$ tunneling. It was also shown there that the splitting between the tunneling levels is identical in the ground $(\mathbf{V}=0)$ and excited $(\mathrm{V}=1)$ states.

In Fig. 3(b) the decomposition into three Lorentzian bands is shown for the observed slightly asymmetric $T_{2 g}$ Raman spectrum of the $\mathrm{OH}^{-}$in $\mathrm{LiF}$ at $6.2 \mathrm{~K}$. The separation and relative strength of the three bands yield the same tunneling splitting parameter $\Delta=1.2 \mathrm{~cm}^{-1}$ and imply that the levels are equidistant. This observation verifies again that the $70^{\circ}$ tunneling is predominant compared to the $110^{\circ}$ and $180^{\circ}$ ones. The half-width $3.7 \mathrm{~cm}^{-1}$ of each Lorentzian band is the same as was obtained from the IR SM absorption (see Table I of Ref. 7). It is close to the observed HW of the $A_{1 g}$ Raman spectrum, which should not contain any unresolved tunneling splitting.

A spectral substructure due to tunneling is also detected in the $\mathrm{KF}: \mathrm{OH}^{-}$system, but here an additional central peak is present, that is not predicted by the $70^{\circ}$ tunneling model. There are two possible approaches to an explanation of this peak. In Fig. 4(a) we have chosen to decompose the IR spectrum of $\mathrm{KF}: \mathrm{OH}^{-}$at $4.2 \mathrm{~K}$ into two Lorentzians and one Gaussian, in such way that the Lorentzian components conform closely to a $70^{\circ}$ tunneling model with the equal tunneling splitting of $\mathrm{v}=0$ and $1 \mathrm{SM}$ states, whereas the Gaussian is treated as an additional absorption of other origin. We used a similar decomposition for the $T_{2 g}$ Raman spectrum in Fig. 4(b), where a central Lorentzian is of course expected, 


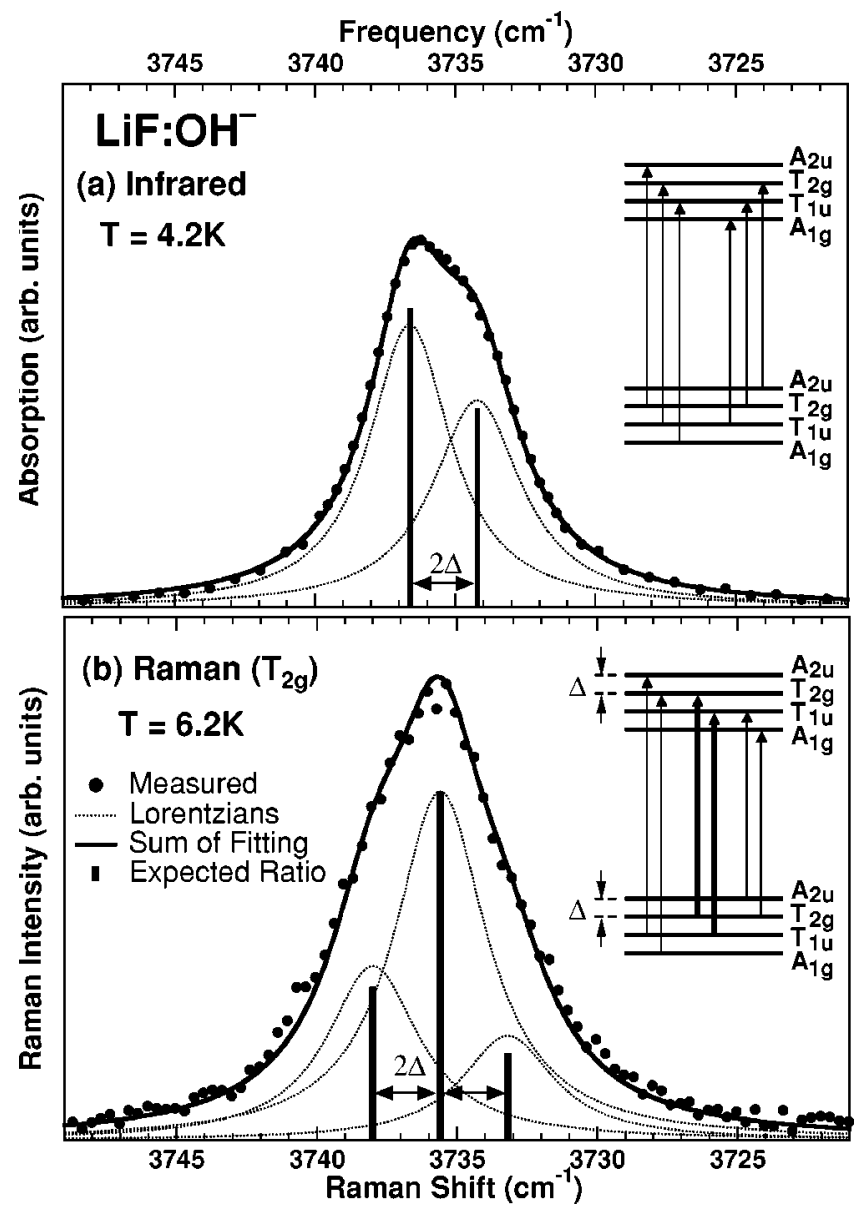

FIG. 3. Infrared (a) and Raman (b) low-temperature SM spectra of $\mathrm{OH}^{-}$in $\mathrm{LiF}$, and their decompositions into Lorentzian-shaped transitions with the positions and relative strength expected for a $\langle 111\rangle$ oriented system with tunneling splitting $\Delta$ (see diagrams in inset).

but a central Gaussian contribution still had to be added to explain the difference between the observed and expected intensity of the central peak. The current fits, as shown on Fig. 4, correspond reasonably to a $70^{\circ}\langle 111\rangle$ reorientational tunneling structure, with an energy-splitting parameter $\Delta$ $=0.6 \mathrm{~cm}^{-1}$. In this approach the nature of the central band can in principle be attributed to a part of the defects for which the tunneling-split vibrational transition has been changed by internal stress or field into a classical $0 \rightarrow 1$ transition. As this could be produced by elastic and electric dipole interactions of the defects, we attempted to remove the central band by reducing the $\mathrm{OH}^{-}$concentration down to 10 ppm, but did not succeed. Background strain from other origins may be an alternative explanation, but it is hard to justify its magnitude. It is interesting to note that our observed behavior of the $\mathrm{OH}^{-}$vibrational transition when changing from $\mathrm{LiF}$ to $\mathrm{KF}$ hosts is amazingly similar to that of the $\left\langle 111\right.$ ) oriented $\mathrm{CN}^{-}$ions under change from $\mathrm{KCl}$ to $\mathrm{KI}$ hosts. ${ }^{17}$ In both cases the reduction of the tunneling splitting from 1.2 to $0.6 \mathrm{~cm}^{-1}$ is accompanied by the appearance of a strong central band. In spite of detailed discussion in Ref. 17, its physical origin remained rather unexplained.

An alternative approach would be to try to decompose both the observed IR absorption and $T_{2 g}$ Raman spectra into only three Lorentzian bands without any Gaussians. The sim- plest tunneling model as in $\mathrm{LiF}: \mathrm{OH}^{-}$system is expanded by including sizable amounts of $110^{\circ}$ and $180^{\circ}$ tunneling probabilities and a difference of the tunneling energy splittings for $v=0$ and $v=1$. Some combination of these effects can produce the triple-band substructure in the $\mathrm{KF}: \mathrm{OH}^{-}$system. However, there are six parameters in this approach. In absence of additional experimental support, we cannot decide what combination is physically correct. Therefore, we plan to perform experiments and quantitative analyses of the SM IR and Raman spectra under stress, in the hope that this will allow us to reach definite conclusions.

The reorientational tunneling of $\mathrm{LiF}: \mathrm{OD}^{-}$and $\mathrm{NaF}: \mathrm{OH}^{-}$ and $\mathrm{OD}^{-}$is not directly observed in either IR or Raman spectra, due to single Lorentzian band shape of the lowtemperature absorption. Besides upper limits of $\Delta$ allowed by the band shapes, lower limits of $\Delta$ values were estimated in Ref. 7 by the small narrowing of the SM absorption under $S_{111}$ stress alignment of the molecules, which removes the $70^{\circ}$ tunneling splitting. From the Raman spectra similar estimates can be made if an unresolved splitting makes the $T_{2 g}$ line wider than the $A_{1 g}$ line. As $\Delta \ll k T$, and the amplitudes of the three components of the $T_{2 g}$ will be $1: 3: 1$, calculated example curves show that the difference between the $A_{1 g}$ and $T_{2 g}$ line width is expected to be between $\Delta$ and $2 \Delta$. For $\mathrm{NaF}: \mathrm{OH}^{-}$this gives a reasonable result: A value of 0.1 to $0.05 \mathrm{~cm}^{-1}$, in good agreement with the IR band-narrowing under stress alignment and with the first results of dielectric measurements on these materials. ${ }^{18}$ For LiF:OD ${ }^{-}$the agreement is not so good, because the observed difference in halfwidth of $\sim 0.8 \mathrm{~cm}^{-1}$, is much larger than the tunneling splitting of $\sim 0.2 \mathrm{~cm}^{-1}$ estimated by IR results. ${ }^{7}$ Note that these Raman linewidths are already close to the instrumental resolution, so that they may contain significant deviations from the real line widths. The measured low-temperature $A_{1 g}$ and $T_{2 g}$ Raman widths of the $\mathrm{NaF}: \mathrm{OD}^{-}$and $\mathrm{KF}: \mathrm{OD}^{-}$lines are meaningless, because the actual values as observed by IR absorption are well below the experimental resolution of the Raman instrument.

\section{Low-temperature linewidths}

If the temperature is varied from $4 \mathrm{~K}$ to $30 \mathrm{~K}$ the widths of the $A_{1 g}$ lines remain approximately constant, with a Lorentzian shape indicating homogeneous broadening. In the case of $\mathrm{LiF}: \mathrm{OH}^{-}$, where the IR and $T_{2 g}$ Raman spectra can be decomposed accurately, the individual components of the tunneling structures also show nearly constant widths, and within the experimental accuracy they are identical to the width of the $A_{1 g}$ line.

The widths of the $A_{1 g}$ Raman line vary from $\sim 4 \mathrm{~cm}^{-1}$ in the $\mathrm{LiF}: \mathrm{OH}^{-}$case, to below the spectral resolution for $\mathrm{OD}^{-}$ in $\mathrm{NaF}$ and KF. Especially the width of the LiF:OH ${ }^{-}$absorption is unusually large, compared to that found in other alkali-halide hosts. These absorption values were measured down to very low $\mathrm{OH}^{-}$concentrations $(\leqslant 10 \mathrm{ppm})$ to verify that interactions between $\mathrm{OH}^{-}$ions are not causing any broadening.

The width $\Delta \omega$ of a Lorentzian line corresponds to the decay time of the correlation function, and both the vibrational lifetime $\left(2 T_{1}\right)$ and the dephasing $\left(T_{2}\right)$ contribute to this. Because the width is almost constant below $30 \mathrm{~K}$, it 


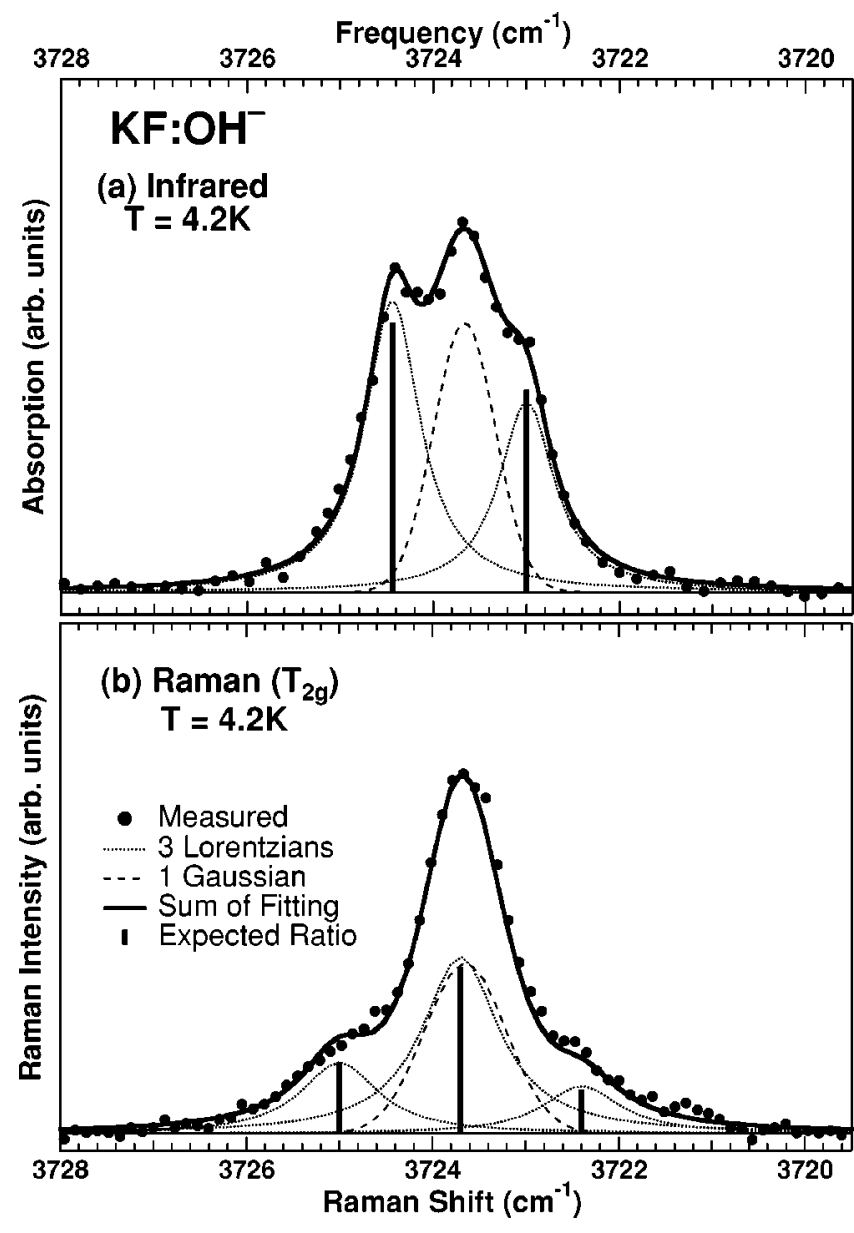

FIG. 4. Infrared (a) and Raman (b) low-temperature SM spectra of $\mathrm{OH}^{-}$in $\mathrm{KF}$, and their decompositions into Lorentzian-shaped "tunneling transitions" and Gaussian-shaped "classical transition", (for details, see text).

seems unlikely that dephasing by phonons or local modes plays an important role in this temperature range. Phononassisted reorientational tunneling ${ }^{19}$ can also be excluded as a dephasing mechanism, for the same reason. On the other hand we observe that the low-temperature half-width is larger if the tunneling splitting is larger. This suggests some relationship. If the level splitting in ground and excited state is not identical, this would cause some apparent line broadening due to hidden splitting; but as no deviation from a Lorentzian shape is visible, this effect cannot be large.

It remains to consider lifetime effects. The observed linewidths correspond to lifetimes of about 1 ps for $\mathrm{LiF}: \mathrm{OH}^{-}, 4$ ps for $\mathrm{LiF}: \mathrm{OD}^{-}, 8$ ps for $\mathrm{KF}: \mathrm{OH}^{-}$, and 9 ps for $\mathrm{NaF}: \mathrm{OH}^{-}$. The other cases are not resolved, but the linewidth is always larger for $\mathrm{OH}^{-}$than for $\mathrm{OD}^{-}$. A lifetime of a few ps for the SM vibration would be extremely short: In other alkali halides the $\mathrm{OH}^{-}$vibration relaxes on a nanosecond time scale. This is already quite fast and has been attributed to a decay of the SM excitation into librational and rotational modes. ${ }^{20}$ This model explains the tendency of the lifetime to remain roughly constant or even increase under $\mathrm{OH}^{-} \rightarrow \mathrm{OD}^{-}$isotope substitution. ${ }^{21}$ Also important in our case is that it is a nearly temperature-independent mechanism, which would be in agreement with the observed temperature independence of the half-width below $30 \mathrm{~K}$. Theoretically the vibrational de- cay could reach the picosecond time scale in favorable conditions. $^{22}$ Favorable conditions require that the librational/rotational mode must be high in frequency and possibly strongly anharmonic, so that the energy of the SM vibration can decay into a minimal number of quanta, and that there is a strong coupling. The coupling may indeed be stronger in the fluorides, because the $\mathrm{OH}^{-}$fits only just into the space of the $\mathrm{F}^{-}$. The librational sidebands of $\mathrm{OH}^{-}$defects in the three hosts are found to be in the range $500-880 \mathrm{~cm}^{-1}$, instead of the frequency of about $300 \mathrm{~cm}^{-1}$ that is common in the alkali chlorides and bromides. ${ }^{23,24}$ The phonon frequencies in the fluorides are also high, and that might assist the fast relaxation.

We tentatively assign the low-temperature width to lifetime broadening, but a direct measurement of the vibrational lifetime would be highly desirable.

\section{Temperature dependence of the half-widths: Dephasing mechanisms}

We have studied the SM vibration of $\mathrm{OH}^{-}$in $\mathrm{LiF}, \mathrm{NaF}$, and $\mathrm{KF}$ hosts under temperature variation, comparing infrared absorption and Raman scattering spectra. The three SM half-widths (full width at half-maximum) of $\mathrm{OH}^{-}$and $\mathrm{OD}^{-}$ in $\mathrm{LiF}, \mathrm{NaF}$, and $\mathrm{KF}$ hosts are plotted in Figs. 5, 6, and 7 as a function of temperature in the range 4-500 K. For simplicity, we plotted the overall half-width, even for $\mathrm{LiF}: \mathrm{OH}^{-}$and $\mathrm{KF}: \mathrm{OH}^{-}$where at low temperature the band could be decomposed into tunneling transitions, as shown in Figs. 3 and 4 at $4 \mathrm{~K}$.

First we focus on the HW[IR] results, measured with higher resolution in a temperature range of $4 \mathrm{~K}$ to $350 \mathrm{~K}$. In contrast to the SM properties of $\mathrm{OH}^{-}$and $\mathrm{OD}^{-}$in alkalichlorides and -bromides, two temperature regions can be distinguished in the behaviors of these systems in the fluorides: Between $4 \mathrm{~K}$ and $50 \mathrm{~K}$ the changes of the half widths are rather small. The overall width of the IR spectra shows some decrease, and for $\mathrm{LiF}: \mathrm{OH}^{-}$this was studied in detail. By decomposing the spectra it was found that as the temperature is increased the linewidths of the individual components become slightly larger, but the tunneling splitting is gradually reduced. The overall effect is a decrease in the total width. From about $50 \mathrm{~K}$ broadening sets in, and above $100 \mathrm{~K}$ the HW[IR] values increase with a $T^{\sim 2}$ power law.

The Raman spectra have a lower resolution than the IR spectra, so the data are incomplete at low temperature, especially in the case of the very narrow $\mathrm{KF}: \mathrm{OD}^{-}$and $\mathrm{NaF}: \mathrm{OD}^{-}$ bands. The effect apparent for $\mathrm{NaF}: \mathrm{OH}^{-}$and $\mathrm{LiF}: \mathrm{OD}^{-}$, where the $A_{1 g}$ Raman line appears to be wider than the absorption line, is caused by the better resolution of the absorption spectrometer. Where measurement is possible, the two Raman HW's show very different temperature dependences: The $\mathrm{HW}\left[T_{2 g}\right]$ is quite similar to the $\mathrm{HW}[\mathrm{IR}]$, but slightly larger. In contrast, the $\mathrm{HW}\left[A_{1 g}\right]$ remains fairly constant up to a higher temperature and thereafter broadens slower. The $\mathrm{HW}\left[A_{1 g}\right]$ then also follows a $T^{\sim 2}$ power law.

At high temperature the $A_{1 g}$ Raman bands are considerably narrower than the $T_{2 g}$ and absorption bands. The difference is probably best explained by looking at the analogous case of molecules dissolved in liquids. ${ }^{25-27}$ In that case the Raman spectrum can be separated into isotropic and aniso- 


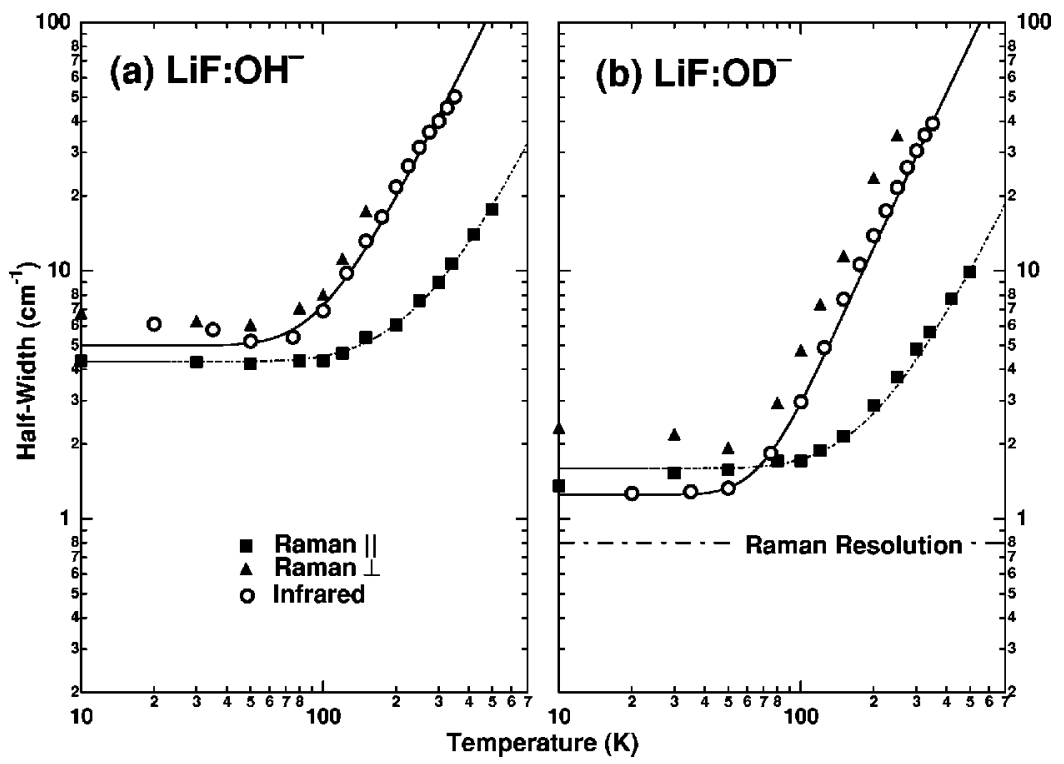

FIG. 5. Temperature dependence of the SM half-widths of $\mathrm{OH}^{-}$and $\mathrm{OD}^{-}$defects in $\mathrm{LiF}$ measured by infrared absorption and by $\|$ and $\perp$ polarized Raman scattering. The lines represent the theoretical formulas, as discussed in the text.

tropic parts: The isotropic Raman spectrum is related to the trace of the polarizability tensor, the anisotropic spectrum to the off-diagonal elements. Dephasing occurs through collisions with the solvent molecules, and the band width of the isotropic Raman spectrum does not contain broadening due to reorientational processes. The anisotropic Raman spectrum and the absorption spectrum do. A review of the attempts that have been made to calculate the actual line shapes for molecules in liquids is given in Ref. 27.

In our case we are dealing with a different situation, that of molecule with a strongly localized orientation in a cubic crystal, and dephasing occurs through collisions with phonons. The system should not be treated as a molecule rotating in a matrix, instead the movements of the molecule and the neighboring atoms should be considered together. Nevertheless a similar interpretation seems suitable: The evolution of the $A_{1 g}$ line shape corresponds to the "pure", or isotropic dephasing of the stretch mode vibration, while the absorption linewidth and the $T_{2 g}$ Raman width also contain the influence of the oscillations of the molecule around its $\langle 111\rangle$ orientation, stimulated by a local mode or by collisions with phonons. The Raman spectra associated with such movements have $E_{g}$ or $T_{2 g}$ symmetry. We were unable to observe the corresponding sidebands in Raman spectra. They have been reported in FTIR absorption spectra. ${ }^{23}$

Some information about the nature of the process can be inferred from the similarity of the absorption and $T_{2 g}$ Raman widths, except in the case of $\mathrm{KF}: \mathrm{OH}^{-}$where the difference is fairly important. It has been calculated in Ref. 28 that for a spherically symmetric molecule in a liquid, an equality of the two linewidths implies that the mean angle of rotation due to collisions is $120^{\circ}$ or larger. The two systems are of course quite different in that in our case only limited movements around the eight orientations, or full reorientations are possible instead of a continuum of angles. But we may perhaps infer from it that the motion of the $\mathrm{OH}^{-}$impurity is "jerky", rather than diffusive, with fairly large amplitudes.

We do not think that the broadening process should be described as a simple rotation or even a libration of the impurity in the lattice, because the frequencies of the librational modes in the alkali fluorides are too high $^{23}$ to correspond to the observed broadening and the SM vibration bands do not

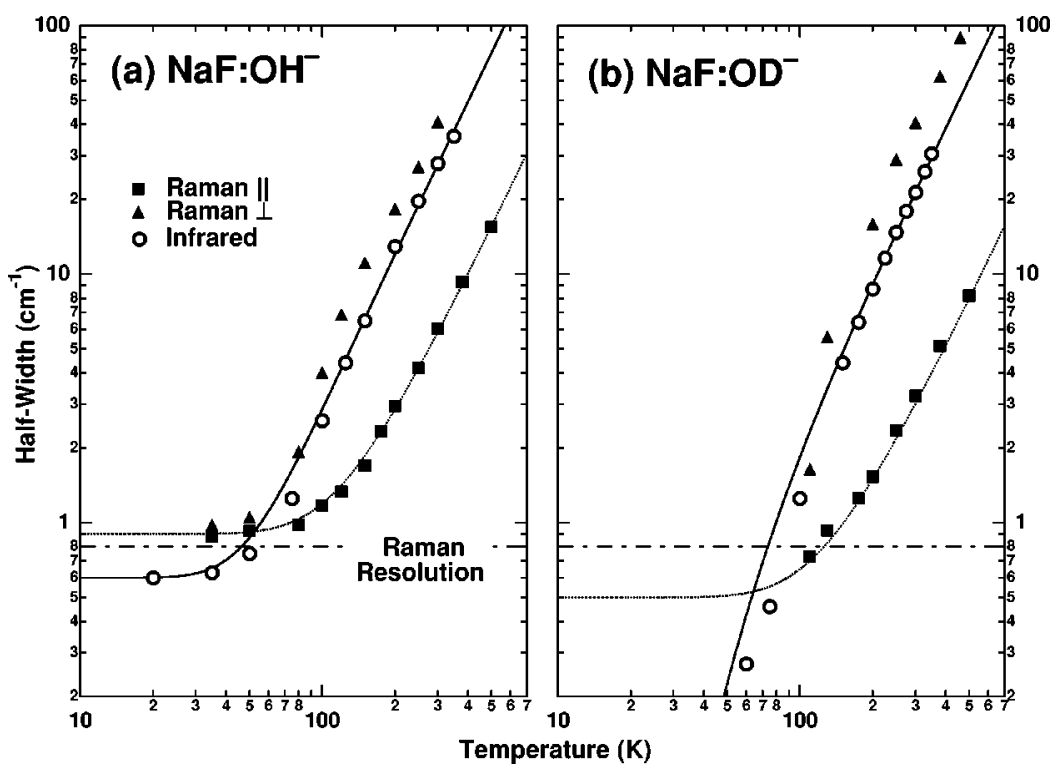

FIG. 6. Temperature dependence of the SM half-widths of $\mathrm{OH}^{-}$and $\mathrm{OD}^{-}$defects in $\mathrm{NaF}$ measured by infrared absorption and by $\|$ and $\perp$ polarized Raman scattering. The lines represent the theoretical formulas, as discussed in the text. 


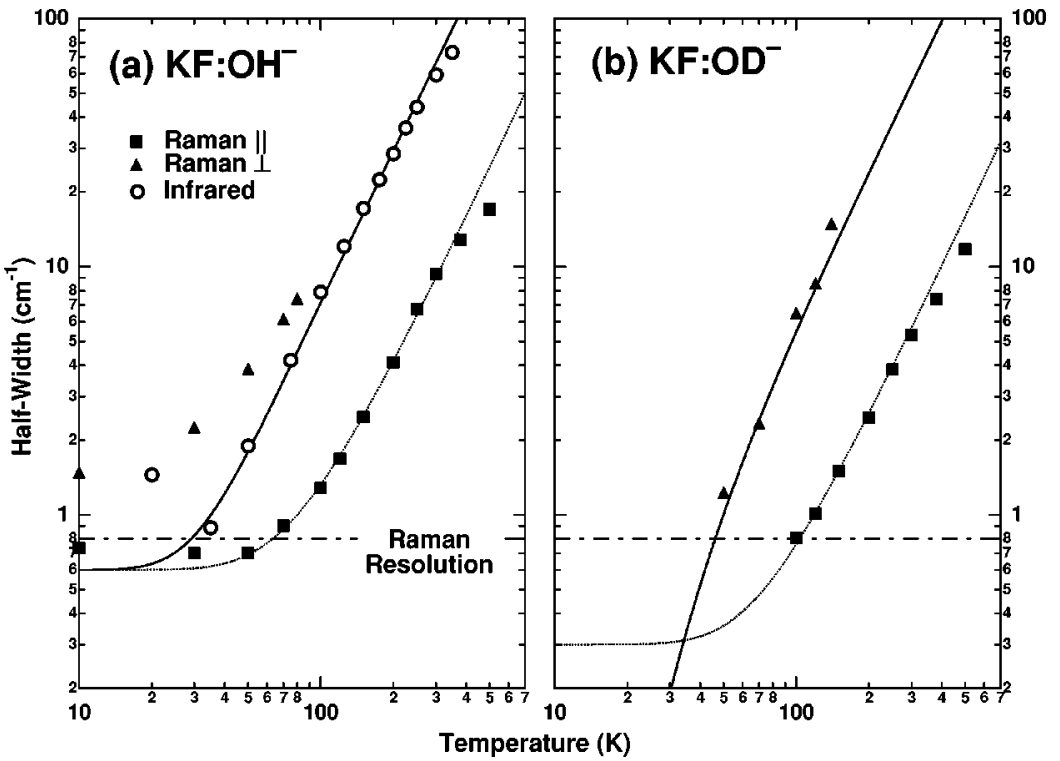

FIG. 7. Temperature dependence of the SM half-widths of $\mathrm{OH}^{-}$and $\mathrm{OD}^{-}$defects in KF measured by infrared absorption and by $\|$ and $\perp$ polarized Raman scattering. The lines represent the theoretical formulas, as discussed in the text.

show any sign of a rotational substructure. However, phonon and local modes may distort the environment of the system, lowering its symmetry, and producing a temporary deviation of the axis of the molecule away from the $\langle 111\rangle$ direction. It is possible that they would greatly enhance the reorientation process.

We have chosen to fit the linewidths in two stages. The temperature-dependence of the "pure" or isotropic dephasing of the $A_{1 g}$ Raman spectrum we fitted with a model for dephasing by weakly-coupled acoustical phonons, ${ }^{29}$ which gives for the line broadening

$$
H W(T)=H W(0)+\omega_{D} \frac{9 W_{D}^{2}}{4}\left(\frac{T}{T_{D}}\right)^{7} \int_{0}^{T_{D} / T} d x \frac{x^{6} e^{x}}{\left(e^{x}-1\right)^{2}}
$$

and the line shift

$$
\omega(T)=\omega_{0}+\omega_{D} \frac{3 W_{D}}{4 \pi}\left(\frac{T}{T_{D}}\right)^{4} \int_{0}^{T_{D} / T} d x \frac{x^{3}}{\left(e^{x}-1\right)} .
$$

These formulas describe the effect of acoustical phonons of frequency $\omega_{D}$, corresponding with a Debye temperature $T_{D}=\hbar \omega_{D} / k$, weakly coupled to the transition with a coupling strength $W_{D}$. For temperatures high compared to $T_{D}$, they predict that the broadening is quadratic with the temperature and the shift linear; which corresponds fairly well to our case. Theoretically, these expressions are nearly exact for $W_{D} \ll T_{D} / T$. However, they remain approximately valid for the parameters we use, as shown in Ref. 29.

Because the Debye approximation is a very simple one and only a part of the phonons are expected to contribute to the dephasing, we have treated $\omega_{D}$ as an adjustable parameter instead of as a constant for the host material. Values for $\omega_{D}$ were chosen that are reasonable for acoustic phonons ${ }^{30}$ and correspond to absorption sidebands of the SM vibration. ${ }^{23} \mathrm{~A}$ fit of this component can also be made assuming dephasing by optical phonons, but the phonon frequencies produced are too low to be realistic for an optical phonon, so we reject these as physically invalid. The parameters for the fits are listed in Table I, and the comparison between the theoretical curve and the data points in Figs. 5, 6, and 7. The sign of the coupling parameter $W_{D}$ cannot be deter-

\begin{tabular}{|c|c|c|c|c|c|c|}
\hline & \multicolumn{2}{|c|}{$\mathrm{LiF}$} & \multicolumn{2}{|c|}{$\mathrm{NaF}$} & \multicolumn{2}{|c|}{$\mathrm{KF}$} \\
\hline & $\mathrm{OH}^{-}$ & $\mathrm{OD}^{-}$ & $\mathrm{OH}^{-}$ & $\mathrm{OD}^{-}$ & $\mathrm{OH}^{-}$ & $\mathrm{OD}^{-}$ \\
\hline \multicolumn{7}{|c|}{ Isotropic component } \\
\hline$\omega_{D}$ & \multicolumn{2}{|c|}{$320 \mathrm{~cm}^{-1}$} & \multicolumn{2}{|c|}{$250 \mathrm{~cm}^{-1}$} & \multicolumn{2}{|c|}{$150 \mathrm{~cm}^{-1}$} \\
\hline$\left|W_{D}\right|$ & 0.263 & 0.204 & 0.260 & 0.198 & 0.255 & 0.204 \\
\hline \multicolumn{7}{|c|}{ Anisotropic component } \\
\hline$\omega_{o}$ & \multicolumn{2}{|c|}{$200 \mathrm{~cm}^{-1}$} & \multicolumn{2}{|c|}{$120 \mathrm{~cm}^{-1}$} & \multicolumn{2}{|c|}{$76 \mathrm{~cm}^{-1}$} \\
\hline$\left|W_{o}\right|$ & 0.326 & 0.286 & 0.204 & 0.189 & 0.256 & 0.239 \\
\hline \multicolumn{7}{|c|}{ Host Parameters } \\
\hline$T_{D}$ & \multicolumn{2}{|c|}{$736 \mathrm{~K}$} & \multicolumn{2}{|c|}{$492 \mathrm{~K}$} & \multicolumn{2}{|c|}{$330 \mathrm{~K}$} \\
\hline Highest LA & \multicolumn{2}{|c|}{$400 \mathrm{~cm}^{-1}$} & \multicolumn{2}{|c|}{$300 \mathrm{~cm}^{-1}$} & \multicolumn{2}{|c|}{$200 \mathrm{~cm}^{-1}$} \\
\hline Phonon & \multicolumn{2}{|c|}{ at $[111]$} & \multicolumn{2}{|c|}{ at $[111]$} & \multicolumn{2}{|c|}{ at $[111]$} \\
\hline
\end{tabular}

TABLE I. Fitting parameters for dephasing by weakly coupled acoustic or optical phonons. 


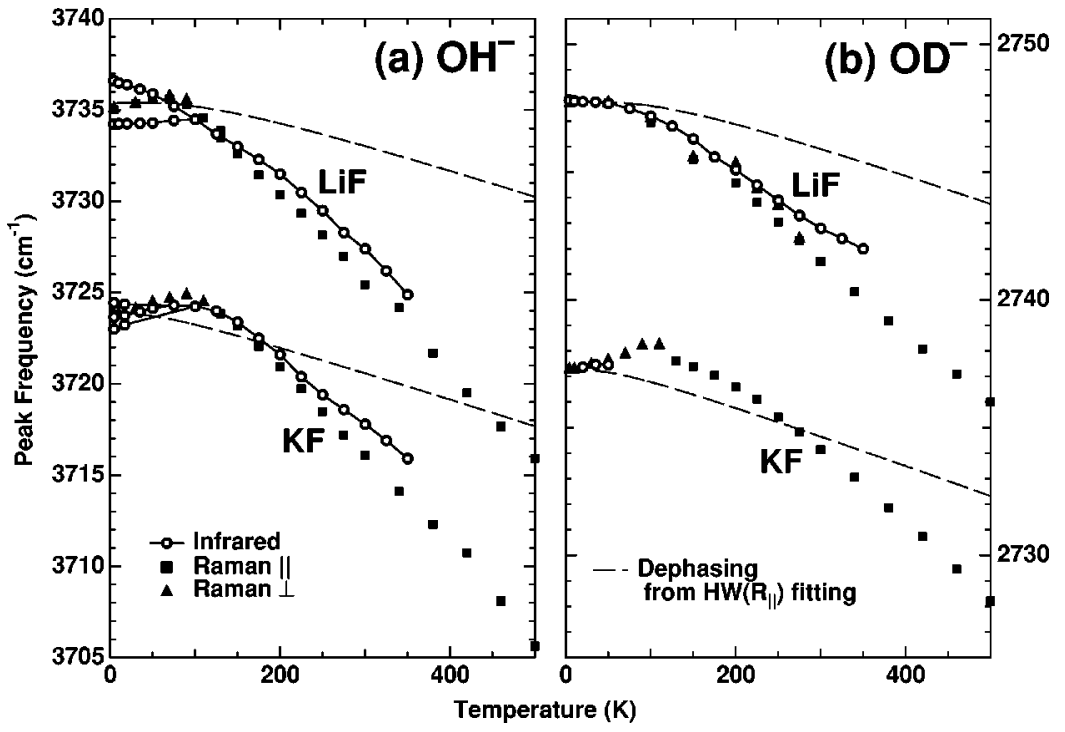

FIG. 8. Frequency of the $\mathrm{OH}^{-}$and $\mathrm{OD}^{-}$ stretching modes in $\mathrm{LiF}$ and $\mathrm{KF}$ as a function of temperature. mined. The values of $\left|W_{D}\right|$ are very similar in all three hosts, and the ratio of the coupling constant for $\mathrm{OH}^{-}$to that for $\mathrm{OD}^{-}$is approximately 1.3 . This stronger coupling for the $\mathrm{OH}^{-}$might simply be caused by the larger amplitude of its vibration.

The difference between the IR or $T_{2 g}$ Raman linewidths and the $A_{1 g}$ Raman line widths we interpreted as an "anisotropic" dephasing. For lack of a better model, we have chosen to treat this as dephasing by optical phonons. A theoretical expression for dephasing by weakly coupled optical phonons is ${ }^{29}$

$$
H W(T)=H W(0)+\omega_{o} \frac{W^{2}}{V} \frac{3}{20} n\left(\omega_{o}\right)\left[n\left(\omega_{o}\right)+1\right] .
$$

This too will produce, in the weak coupling limit and at high temperatures, a quadratic broadening of the band. We chose the approximation for optical phonons because of the narrowness of the sideband, which resemble an optical phonon band more than an acoustical phonon band. For the relative width of the band we chose $V=0.1$.

As effective frequencies $\omega_{o}$ we selected some that correspond to sidebands of the SM vibration that were shown by elastic stress experiments to be a transverse mode. ${ }^{23} \mathrm{We}$ tried several of the present bands, to find the best fit. In the case of $\mathrm{KF}$ we were forced to use a frequency of about $75 \mathrm{~cm}^{-1}$, corresponding to a region where the sideband absorption is weak. The parameters are again listed in Table I, and the curves corresponding to the sum of the two dephasing components are plotted on Figs. 5, 6, and 7. The coupling constant is largest in $\mathrm{LiF}$ and smallest in $\mathrm{NaF}$. The ratio between the coupling constants $\left|W_{o}\right|$ for $\mathrm{OH}^{-}$and $\mathrm{OD}^{-}$is about 1.1, a smaller difference than was found for $\left|W_{D}\right|$. This seems reasonable enough if we attribute the anisotropic dephasing largely to movements of the nearest-neighbor ions, coupled to the orientation of the impurity molecule. These movements can be expected to have a reduced isotopedependence. Likewise, the center-of-mass motion of the impurity should be little influenced by the isotope substitution.

A good agreement is found between the fit curves and the data points, but the importance of this should not be exag- gerated. It only shows that a reasonable fit is possible with a very simple model. Indeed this turned out to be possible with a wide variation of parameters, so we had to make some essentially arbitrary assumptions about the frequencies $\omega_{D}$ and $\omega_{o}$.

\section{E. Temperature dependence of the SM peak positions}

The frequencies of the SM vibration peak in $\mathrm{LiF}$ and $\mathrm{KF}$ are plotted in Fig. 8. At low temperature the tunneling structure for $\mathrm{LiF}: \mathrm{OH}^{-}$is resolved, and the absorption band clearly has two peaks. The positions of these two peaks are plotted at the lowest temperatures, with the position of the main Raman line between them. With increasing temperature, the tunneling splitting soon vanishes. In the case of $\mathrm{KF}: \mathrm{OD}^{-}$there is a "classical" transition with a Gaussian line shape between the two tunneling peaks, so that the band has a single highest maximum. We indicate this on the figure. The KF positions also appear to shift to higher frequencies over the first $100 \mathrm{~K}$.

The SM frequencies all have in common (a) that they shift to lower energies above $100 \mathrm{~K}$, (b) that the shift over the temperature range $4 \mathrm{~K}-350 \mathrm{~K}$ is relatively large $\left(8-11 \mathrm{~cm}^{-1}\right)$ compared to that in most other alkali halides (e.g. $\approx 5 \mathrm{~cm}^{-1}$ for $\mathrm{KBr}: \mathrm{OH}^{-}$), and (c) that the $A_{1 g}$ Raman line, which can be followed to high temperatures, shifts a little more than the absorption band. Above $100 \mathrm{~K}$, the difference increases approximately linearly with temperature.

At high concentrations, above $1000 \mathrm{ppm}$, there is a shift of the $A_{1 g}$ and $T_{2 g}$ Raman lines relative to each other, of about $2 \mathrm{~cm}^{-1}$ for $\mathrm{NaF}: \mathrm{OD}^{-}$and $1 \mathrm{~cm}^{-1}$ for $\mathrm{LiF}: \mathrm{OD}^{-}$. We did not include these data in Fig. 8. This difference could be caused by interactions between the impurities, which result in combination modes of various symmetry with slightly different frequencies. However, the half-widths of the lines are not different from those in crystals with lower concentrations.

Several factors will contribute to the temperature dependence of the peak position. The dephasing processes also influence the observed frequency of the stretch mode vibrations, as described by Eq. (3.2). There is only a small difference between the positions of the IR and $A_{1 g}$ Raman lines, 
TABLE II. Absolute IR absorption intensities $A$ and relative Raman intensities $I$ per $1000 \mathrm{ppm}$ of $\mathrm{OH}^{-}$ and $\mathrm{OD}^{-}$, measured in $\mathrm{LiF}, \mathrm{NaF}$, and KF. From the measurements of $A$, values of $(\partial p / \partial r)^{2}$ are obtained directly. From the relative Raman measurements of $I$, only the relative ratio of $(\partial \bar{\alpha} / \partial r)^{2}$ between $\mathrm{OH}^{-}$and $\mathrm{OD}^{-}$can be obtained, using appropriate frequency and reduced mass corrections as indicated in the text.

\begin{tabular}{|c|c|c|c|c|}
\hline & & $\mathrm{LiF}$ & $\mathrm{NaF}$ & $\mathrm{KF}$ \\
\hline \multirow{5}{*}{ IR } & $A_{\mathrm{OH}^{-}}$in $\mathrm{cm}^{-2} / 1000 \mathrm{ppm}$ & 300 & 60 & 7.4 \\
\hline & $A_{\mathrm{OD}^{-}}$in $\mathrm{cm}^{-2} / 1000 \mathrm{ppm}$ & 300 & 70 & 0.02 \\
\hline & $(\partial p / \partial r)_{\mathrm{OH}^{-}}^{2}$ in $10^{-3} e^{2}$ & 24 & 7.4 & 1.4 \\
\hline & $(\partial p / \partial r)_{\mathrm{OD}^{-}}^{2}$ in $10^{-3} e^{2}$ & 45 & 16 & 0.007 \\
\hline & $(\partial p / \partial r)^{2}$ ratio for $\mathrm{OH}^{-}$to $\mathrm{OD}^{-}$ & 0.53 & 0.46 & 200 \\
\hline \multirow{3}{*}{ Raman } & $I_{\mathrm{OH}^{-}}$in arb. units & 41 & 78 & 24 \\
\hline & $I_{\mathrm{OD}^{-}}$in arb. units & 86 & 116 & 29 \\
\hline & $(\partial \bar{\alpha} / \partial r)^{2}$ ratio for $\mathrm{OH}^{-}$to $\mathrm{OD}^{-}$ & 0.46 & 0.66 & 0.81 \\
\hline
\end{tabular}

although the difference in linewidth is large. One would expect the pure or isotropic dephasing to produce, if not all, then at least most of the frequency shift. The predicted frequency shift due to isotropic dephasing is indicated on Fig. 8. It is clearly far too small to reproduce all of the temperature dependence we observe.

Thermal lattice expansion will in general reduce the frequency of the stretch mode by moving the nearest neighbors outside, so that the molecule feels a smaller repulsive potential. Variations of host material confirm the natural assumption, that the frequency of the SM will be higher if the molecule is confined in a small cavity and lower if it has more space. Compared with the frequency shifts, the thermal expansion has roughly the correct shape: It is approximately linear above $200 \mathrm{~K}^{31}$ If the variation of frequency vs anion diameter $^{32}$ is extrapolated from one host to another, ${ }^{33}$ and this used to estimate the effect of the thermal expansion, then the expected reduction of the frequency from $0 \mathrm{~K}$ to $500 \mathrm{~K}$ is about $5 \mathrm{~cm}^{-1}$ for $\mathrm{OH}^{-}$and $3 \mathrm{~cm}^{-1}$ for $\mathrm{OD}^{-}$. Again, this is too small to explain the observed frequency shift. But the details of the interaction between the impurity and its neighbors are unknown, and it is very well possible that our estimate of the effect of thermal expansion is too low. Measurements of the harmonics of the $\mathrm{OH}^{-}$and $\mathrm{OD}^{-}$absorption have shown that a Morse potential is a very good model for the mechanical behavior of the diatomic molecular defects in alkali halides, but that there are small deviations in the fluorides, possibly because of a weak bonding between the hydrogen atom and the neighboring ions. ${ }^{33}$

\section{F. Intensity of IR and Raman SM transitions}

Besides the half-widths and positions of the $\mathrm{OH}^{-}$and $\mathrm{OD}^{-}$SM transitions, discussed in Secs. III D and III E, our spectral IR and Raman measurements contain information about their strength under host and isotope variations. Temperature variation can be neglected because we find, within experimental accuracy, no temperature dependence for both Raman and IR absorption strength.

In Table II we summarize for $\mathrm{OH}^{-}$and $\mathrm{OD}^{-}$in all three hosts the measured and derived quantities, which are significant for the studied SM transitions. Integrated IR absorption measurements, performed at any temperature in samples of well-determined concentration $N$, yield reliable absolute values $A$ in normalized units of $\mathrm{cm}^{-2} / 1000 \mathrm{ppm}$. These $A / N$ quantities, multiplied by essentially a constant and the reduced mass $\mu$ of the molecule, yield the important values of $(\partial p / \partial r)^{2}$, where $p$ is the molecular dipole moment with respect to the center of mass and $r$ the internuclear distance. ${ }^{34}$

The second part of Table II summarizes the quantities related to Raman strength. We measured this only relatively, e.g., for $\mathrm{OH}^{-}$in the three hosts by different runs in samples of known values $N$ under equal power excitation of $\lambda_{\text {exc }}$ $=514.5 \mathrm{~nm}$ and best possible equal optical alignment. This yields (still with some uncertainty) the values $I_{\mathrm{OH}^{-}}$in units of photon counts per second normalized to $N=1000 \mathrm{ppm}$. The $I_{\mathrm{OD}^{-}}$values, relative to $I_{\mathrm{OH}^{-}}$, we obtain accurately by measuring for each host a specially grown sample double doped with the same concentration of $\mathrm{OH}^{-}$and $\mathrm{OD}^{-}$. Similar as in IR absorption, the measured Raman intensities $I$ multiplied by the molecular reduced mass $\mu$ and a "frequency-correction factor' $C$ (Ref. 35) yield values of $(\partial \bar{\alpha} / \partial r)^{2}$, with $\bar{\alpha}$ the electronic polarizability of the molecule. As our Raman intensities are not absolutely calibrated but only measured as relative values, we obtain from them only the relative $(\partial \bar{\alpha} / \partial r)^{2}$ ratio between our different systems.

When comparing the same molecules in the three hosts, their $\mu$ value and correction factor is the same for all, and the intensity ratios reflect directly the ratios of $(\partial \bar{\alpha} / \partial r)^{2}$. When comparing $\mathrm{OH}^{-}$and $\mathrm{OD}^{-}$of different $\mu$ and $\omega_{S M}$, the ratio $C_{\mathrm{OH}^{-}} / C_{\mathrm{OD}^{-}}$is close to nearly exactly compensated by that of $\mu_{\mathrm{OH}^{-}} / \mu_{\mathrm{OD}^{-}}$, so that in total a correction factor close to $1(\sim 1.1)$ results. Therefore, essentially the measured Raman intensity ratio $I_{\mathrm{OH}^{-}} / I_{\mathrm{OD}^{-}}$reflects nearly exactly the $(\partial \bar{\alpha} / \partial r)^{2}$ ratio of $\mathrm{OH}^{-}$and $\mathrm{OD}^{-}$, as summarized in Table II.

First discussing the IR absorption strength, we recognize two important trends and one "singularity" in the $(\partial p / \partial r)^{2}$ values listed in Table II.

(a) Under $\mathrm{LiF} \rightarrow \mathrm{NaF} \rightarrow \mathrm{KF}$ exchange of hosts, a strong systematic decrease (by a factor up to 40) in the values for $\mathrm{OH}^{-}$.

(b) Under $\mathrm{OH}^{-} \rightarrow \mathrm{OD}^{-}$isotope exchange in $\mathrm{LiF}$ and $\mathrm{NaF}$, an increase of $(\partial p / \partial r)^{2}$ by a factor of $\sim 2$. 


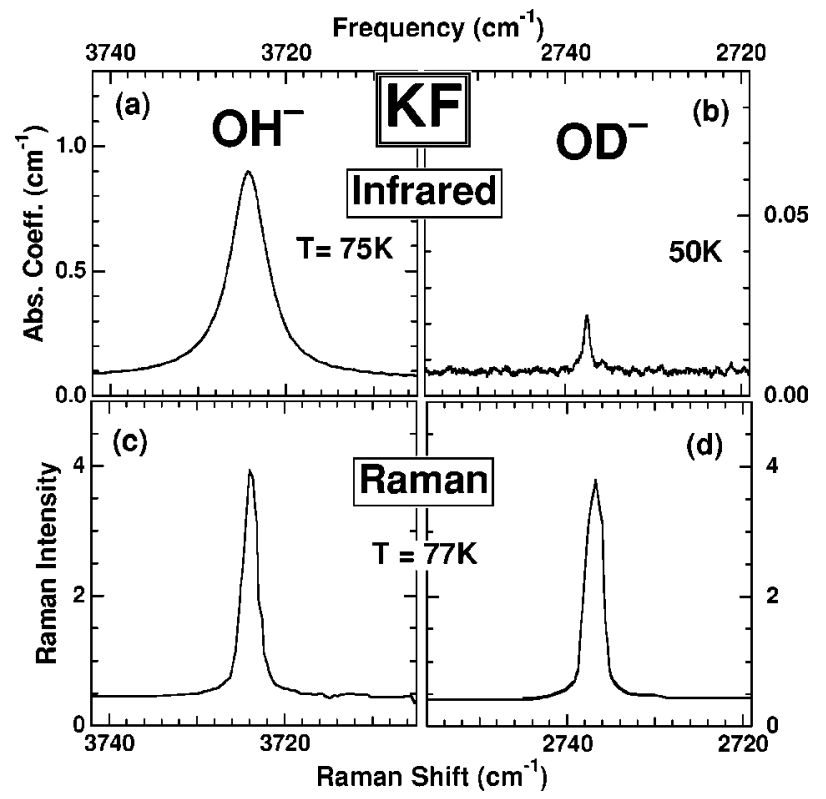

FIG. 9. SM spectra at medium temperatures of KF with equal concentrations of $\mathrm{OH}^{-}$and $\mathrm{OD}^{-}$defects: (a) and (b) are infrared absorption, plotted for $\mathrm{OH}^{-}$and $\mathrm{OD}^{-}$in very different absorption scales. (c) and (d) are Raman scattering, plotted for $\mathrm{OH}^{-}$and $\mathrm{OD}^{-}$ in the same scale of scattering intensity. The scattering efficiency and detection efficiency factors reduce the $\mathrm{OH}^{-}$relative strength by a factor 0.75 .

(c) Under the same isotope exchange in KF, the "singularity" of a drop of $(\partial p / \partial r)^{2}$ by a huge factor nearly down to zero. This is illustrated by the IR spectra in Figs. 9(a) and 9(b), plotted in very different scales for $\mathrm{OH}^{-}$and $\mathrm{OD}^{-}$.

For understanding and interpretation of these results, we consider three significant aspects:

(A) By substitution of $\mathrm{OH}^{-}$into various hosts, the internuclear distance $r_{e}$ of the free molecule will be reduced to smaller values (most in $\mathrm{LiF}$ ) due to the compression along the molecular axis by repulsive interaction with its nearestneighboring host cations.

(B) For $\mathrm{OH}^{-} \rightarrow \mathrm{OD}^{-}$isotope exchange, only a very small reduction of $r_{e}$ will occur (due to the anharmonicity of the Morse-type mechanical SM potential). Much more significant is the isotopic shift of the molecular center of mass $(\mathrm{CM})$, which makes the $(\partial p / \partial r)$ of $\mathrm{OD}^{-}$larger by $\sim 0.05|e|$ than that of $\mathrm{OH}^{-}$. $^{36}$

(C) As shown by quantum chemical calculations, ${ }^{37}$ the electric dipole function $p(r)$ of $\mathrm{OH}^{-}$is a complicated one, approximately described by a "Sage function', $p(r)$ $\propto r \cdot \exp \left(-r / r^{*}\right)$, with $r^{*}$ an adjustable parameter. ${ }^{38}$ Evidently, its positive slope at $r<r^{*}$ decreases with rising $r$, becomes zero at $r=r^{*}$, and then negative for $r>r^{*}$. For $\mathrm{OH}^{-}$as a free molecule or substituted into larger lattices (like $\mathrm{KCl}$ and $\mathrm{KBr}), r_{e}>r^{*}$ and $(\partial p / \partial r)$ is negative. This is proven experimentally by the observation that the small positive increase of $(\partial p / \partial r)$ by the $\mathrm{CM}$ shift under $\mathrm{OH}^{-}$ $\rightarrow \mathrm{OD}^{-}$substitution [discussed in (B)] leads to decrease in magnitude of $|\partial p / \partial r|$ and its squared $(\partial p / \partial r)^{2}$, as observed in $\mathrm{KCl}$ and $\mathrm{KBr}$. ${ }^{34}$

We interpret our results (a), (b), and (c) on the basis of these aspects (A), (B), and (C). For $\mathrm{LiF}$ and $\mathrm{NaF}$ we assume $\mathrm{OH}^{-}$and $\mathrm{OD}^{-}$to lie at $r_{e}<r^{*}$ and to have positive $(\partial p / \partial r)$.
The best proof for this is the observation (b), that the CM shift by $\mathrm{OH}^{-} \rightarrow \mathrm{OD}^{-}$substitution leads to a net increase of $(\partial p / \partial r)^{2}$, opposite to its decrease in larger lattices like $\mathrm{KCl}$ and $\mathrm{KBr}^{34}$ In between these two types of small and large lattices with opposite $\mathrm{OH}^{-} / \mathrm{OD}^{-}$behavior lies the interesting region of $r_{e} \approx r^{*}$, with small $|\partial p / \partial r|$. Following the LiF $\rightarrow \mathrm{NaF}$ trend, KF lies in this range with $r_{e}$ slightly above $r^{*}$ for $\mathrm{OH}^{-}$, with a $(\partial p / \partial r)$ close to $-0.05|e|$. Its small $(\partial p / \partial r)^{2}$ value becomes reduced to nearly zero by the CM shift under $\mathrm{OH}^{-} \rightarrow \mathrm{OD}^{-}$isotope exchange. A very similar behavior of $\mathrm{OH}^{-} / \mathrm{OD}^{-}$absorption strength is observed in the $\mathrm{NaCl}$ host, ${ }^{34}$ which has a similar lattice constant as KF. When the slope of the dipole function $p(r)$ disappears at $r$ $=r^{*}$, its second derivative $\partial^{2} p / \partial r^{2}$ is very high; as a consequence we observe for $\mathrm{OD}^{-}$in both $\mathrm{KF}$ and $\mathrm{NaCl}$ hosts a second harmonic SM absorption stronger than the (nearly disappeared) first harmonic. ${ }^{33}$

Our results on the Raman intensity and $(\partial \bar{\alpha} / \partial r)^{2}$ variations in the six systems are quite different, and simpler. Both host variation (measurable only with larger error-bar) and $\mathrm{OH}^{-} \rightarrow \mathrm{OD}^{-}$isotope exchange keep the $(\partial \bar{\alpha} / \partial r)^{2}$ values rather constant. Obviously, the "polarizability function" $\bar{\alpha}(r)$ (which we did not find calculated in the literature), keeps its slope $(\partial \bar{\alpha} / \partial r)$ rather constant over the range of our $r_{e}$ variation. The only small trend we observe is the relative Raman strength of $\mathrm{OH}^{-}$and $\mathrm{OD}^{-}$, which is higher for $\mathrm{OD}^{-}$ in $\mathrm{LiF}$ and decreases via $\mathrm{NaF}$ towards $\mathrm{KF}$ to nearly equal strength. This latter case of KF is in fact illustrated in Figs. 9(c) and 9(d) by its measured $\mathrm{OH}^{-}$and $\mathrm{OD}^{-}$Raman spectra in KF, emphasizing that the huge drop in IR absorption strength [Figs. 9(a) and 9(b)] is totally absent in Raman.

\section{SUMMARY}

Our spectra confirm that the $\mathrm{OH}^{-}$and $\mathrm{OD}^{-}$centers in $\mathrm{LiF}, \mathrm{NaF}$, and $\mathrm{KF}$ are oriented along the $\langle 111\rangle$ axes of the host crystal. The line shapes remain Lorentzian up to high temperature, indicating that the impurities are well localized in their wells. They orient themselves parallel with the direction in which a uniaxial pressure is applied.

Tunneling splittings of $1.2 \mathrm{~cm}^{-1}$ for $\mathrm{LiF}: \mathrm{OH}^{-}$and $0.7 \mathrm{~cm}^{-1}$ for $\mathrm{KF}: \mathrm{OH}^{-}$can be determined. The difference between the linewidths of the $A_{1 g}$ and $T_{2 g}$ Raman spectra sets an upper limit for the splitting in other hosts. In LiF the spectra correspond very well with those predicted by a model involving nearest-well tunneling. In KF the low-temperature IR and Raman spectra show some anomalies, possibly caused by impurities that do not participate in the tunneling behavior and that are present even at very low concentrations.

The linewidths of the transitions could possibly be explained by assuming that they are lifetime related below 30 $\mathrm{K}$. However, it is desirable to have an independent test of this extremely short vibrational lifetime. At higher temperatures, the absorption and $T_{2 g}$ Raman lines are strongly broadened by interaction with phonons. The $A_{1 g}$ Raman bands are broadened less. We attributed the difference to the presence of "isotropic" and "anisotropic" dephasing components. The latter are due to oscillations of the molecule 
around the $\langle 111\rangle$ axis, and do not contribute to the broadening of the $A_{1 g}$ line.

The temperature dependence of the SM frequency is considerably stronger than we estimated from both the dephasing effect and the thermal expansion of the lattice. It is likely that short-range forces are fairly important for $\mathrm{OH}^{-}$in the alkali-fluorides, and that we underestimated the effect of thermal expansion.

The variation of absorption intensity under host variation and under $\mathrm{OH}^{-} \leftrightarrow \mathrm{OD}^{-}$isotope substitution indicates a strong electrical anharmonicity, in other words the second derivative of the dipole moment is large. It is also concluded that in the case of $\mathrm{OD}^{-}$the dipole moment reaches a maximum (or minimum) as a function of the equilibrium distance in $r_{e}$ in $\mathrm{KF}$ and $\mathrm{NaCl}$. The Raman cross sections show considerably less dependence on the host material and on isotope variation.

\section{ACKNOWLEDGMENTS}

This work was supported by NSF Grant No. DMR 9632959. Support by the FWO (Fund for Scientific Research, Flanders), the IIKW (Interuniversitary Institute for Nuclear Sciences), and the Belgian IIKW (Interuniversitary Institute for Nuclear Sciences), and the Belgian Lotto is gratefully acknowledged. E.G. would like to thank the FWO for financial support.
${ }^{1}$ F. Bridges, CRC Crit. Rev. Solid State Sci. 5, 1 (1975).

${ }^{2}$ H.B. Shore and L.M. Sander, Phys. Rev. B 6, 1551 (1972).

${ }^{3}$ W.E. Bron and R.W. Dreyfus, Phys. Rev. Lett. 16, 165 (1966).

${ }^{4}$ S. Kapphan and J. Koppitz (unpublished).

${ }^{5}$ K. Guckelsberger, J. Phys. Chem. Solids 41, 1209 (1980).

${ }^{6}$ D. Moy, R.C. Potter, and A.C. Anderson, J. Low Temp. Phys. 52, 115 (1983).

${ }^{7}$ C.P. An and F. Luty, Phys. Rev. B 56, R5721 (1997).

${ }^{8}$ H. Fleurent, W. Joosen, and D. Schoemaker, Phys. Rev. B 38, 6257 (1988).

${ }^{9}$ J.G. Peascoe and M.V. Klein, J. Chem. Phys. 59, 2394 (1973).

${ }^{10}$ J.F. Zhou, E. Goovaerts, and D. Schoemaker, Phys. Rev. B 29, 5509 (1984).

${ }^{11}$ J.D. Eshelby, Solid State Phys. 3, 79 (1956).

${ }^{12}$ H. Hätel and F. Luty, Phys. Status Solidi 12, 347 (1965).

${ }^{13}$ M. Gomez, S.P. Bowen, and J.A. Krumhansl, Phys. Rev. 153, 1009 (1967).

${ }^{14}$ D. Durand and F. Luty, Phys. Status Solidi B 81, 443 (1977).

${ }^{15}$ H.U. Beyeler, Phys. Rev. B 10, 2614 (1974).

${ }^{16}$ As both absorptions are a superposition of the same three Stokes or anti-Stokes shifted $g \leftrightarrow u$ transitions between equidistant tunneling states [see inset of Fig. 3(a)], their composites are expected to have at $k T>\Delta$ the same spectral shape or width. The observation of smooth Lorentzian shape for both of them proves equal lifetime of the four states within each multiplet, but does not exclude a possible lifetime difference between the $\mathrm{v}=0$ and $\mathrm{v}=1$ multiplet (e.g., due to the rapid nonradiative relaxation of the excited $v=1$ state).

${ }^{17}$ H.U. Beyeler, Phys. Rev. B 11, 3078 (1975).

18 C. Enss and F. Luty (private communication).

${ }^{19}$ B.G. Dick, Phys. Status Solidi A 29, 587 (1986).
${ }^{20}$ C.E. Mungan, U. Happek, and A.J. Sievers, J. Lumin. 58, 33 (1994).

${ }^{21}$ E. Gustin, M. Leblans, A. Bouwen, D. Schoemaker, and F. Luty, Phys. Rev. B 54, 6963 (1996).

${ }^{22}$ K. Freed and H. Metiu, Chem. Phys. Lett. 48, 262 (1977).

23 C.P. An and F. Luty, Abstract Book of the 8th Europhysical Conference on Defects in Insulating Materials, EURODIM, 1998 (Keele University, UK, 1998), p 144; C.P. An and F. Luty (unpublished).

${ }^{24}$ M.V. Klein, B. Wedding, and M.A. Levine, Phys. Rev. 180, 902 (1969).

${ }^{25}$ E. Brindeau, S. Bratos, and J.C. Leicknam, Phys. Rev. A 6, 2007 (1972).

${ }^{26}$ S. Bratos and E. Marechal, Phys. Rev. A 4, 1078 (1971).

${ }^{27}$ David W. Oxtoby, Adv. Chem. Phys. 40, 1 (1979).

${ }^{28}$ N.E. Ivanov, Sov. Phys. JETP 18, 1041 (1954).

${ }^{29}$ D. Hsu and J.L. Skinner, J. Chem. Phys. 81, 5471 (1984).

${ }^{30} \mathrm{H}$. Bilz and W. Kress, Phonon Dispersion Relations in Insulators (Springer Verlag, Berlin, 1979).

${ }^{31}$ Y.S. Touloukian, R.K. Kirhy, R.E. Taylor, and T.Y.R Lee, Thermal Expansion - Nonmetallic Solids, Thermophysical Properties of Matter (IFI/Plenum, New York, 1977), Vol. 13.

${ }^{32}$ L. Bosi and M. Nimis, Nuovo Cimento D 13, 377 (1991).

${ }^{33}$ A. Afanasiev, C.P. An, and F. Luty, in Defects in Insulating Materials, edited by O. Kanert and J.-M. Spaeth (World Scientific, London, 1993), Vol. 1, p. 551.

${ }^{34}$ B. Wedding and M.V. Klein, Phys. Rev. 177, 1274 (1969).

${ }^{35}$ T.V. Long and R.A. Plane, J. Chem. Phys. 43, 457 (1965).

${ }^{36}$ A.R. Woll and W.B. Fowler, Phys. Rev. B 48, 16788 (1993).

${ }^{37}$ H.J. Werner, P. Rosmus, and E.A. Reinsch, J. Chem. Phys. 79, 905 (1983).

${ }^{38}$ M.L. Sage, Chem. Phys. 35, 375 (1978). 